\title{
Suspected Pterin-4a-carbinolamine Dehydratase Deficiency: Hyperphenylalaninaemia due to Inhibition of Phenylalanine Hydroxylase by Tetrahydro-7-biopterin
}

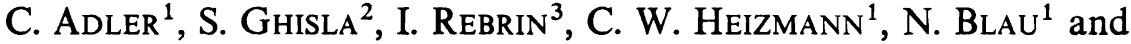 \\ H.-Ch. CurTiUs ${ }^{1 *}$ \\ ${ }^{1}$ Division of Clinical Chemistry, Department of Pediatrics, University of Zurich, \\ Steinwiesstr. 75, CH-8032 Zurich, Switzerland; ${ }^{2}$ Faculty of Biology, University of \\ Konstanz, D-7750 Konstanz, Germany; ${ }^{3}$ Department of Human Genetics, University \\ of Greifswald, D/O-2200 Greifswald, Germany
}

A new form of atypical phenylketonuria coupled with a transient hyperphenylalaninaemia has recently been discovered. The new metabolic disorder is characterized by an accompanying excretion of 7-substituted pterins, i.e. 7-biopterin (primapterin), 6-oxo-7-biopterin and 7-neopterin (anapterin) in the patients' urine (Curtius et al 1988; Dhondt et al 1988). This excretion of 7-substituted pterins appears to be associated with an enzyme defect in the aromatic amino acid hydroxylating system. Formation of 7-pterins was also accomplished in vitro when the phenylalanine hydroxylase reaction (using human or rat enzyme) was carried out with 4a-carbinolamine dehydratase-free preparations. The 7-substituted pterins are formed from the 6substituted ones (Curtius et al 1990a; Davis et al 1991). The conclusion was reached that the patients suffering from this phenylalaninaemia most probably suffer from a carbinolamine dehydratase deficiency.

4a-Hydroxycarbinolamine, an intermediate of the hydroxylation reaction, is relatively stable at alkaline $\mathrm{pH}$ and in the absence of carbinolamine dehydratase. These conditions favour accumulation of 4a-hydroxycarbinolamine in vitro and rearrangement to 7-pterins. The latter most probably is formed via a spiro intermediate (Figure 1).

When D-tetrahydroneopterin was used instead of L-tetrahydrobiopterin $\left(\mathrm{BH}_{4}\right)$ as reduced pterin cofactor in the in vitro experiments carried out with phenylalanine hydroxylase, we observed the formation of the isomer 7-neopterin. Since 7-neopterin excretion was also found in healthy controls as well as in patients with classical and atypical phenylketonuria, we can conclude that tetrahydroneopterin is also a cofactor in vivo of the aromatic amino acid hydroxylation system. In order to be used in this

${ }^{*}$ Correspondence: 


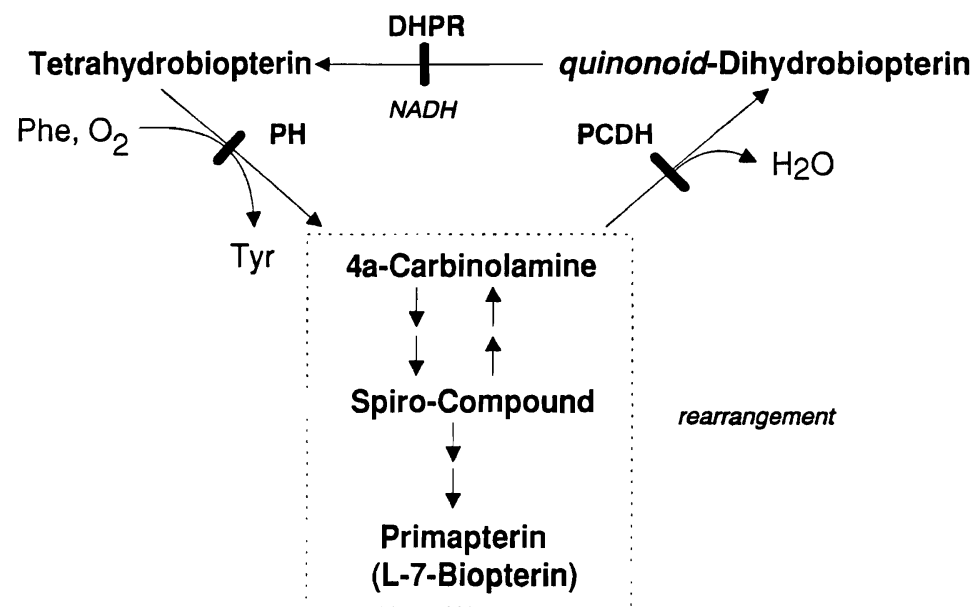

Figure 1 The metabolic pathway of phenylalanine hydroxylase reaction. $\mathrm{PH}=$ phenylalanine hydroxylase. $\mathrm{PCDH}=$ pterin-4a-carbinolamine dehydratase

reaction, dihydroneopterin first has to be formed from dihydroneopterin triphosphate and then must be reduced to tetrahydroneopterin by dihydrofolate reductase.

In this paper we show that the formation of the 7-isomer also occurs when 6hydroxytetrahydromethylpterin is used instead of tetrahydrobiopterin in in vitro incubation experiments with carbinolamine dehydratase-free phenylalanine hydroxylase. In addition, we performed kinetic experiments in order to verify whether and to what extent tetrahydroprimapterin inhibits phenylalanine hydroxylase. A positive finding could provide an explanation for the occurrence of a transient hyperphenylalaninaemia in these patients. In the urine of primapterinuric patients, only $20 \%$ of biopterin and 7-biopterin were excreted in their tetrahydro form (normal controls about $80 \% \mathrm{~L}^{-\mathrm{BH}_{4}}$ of total L-biopterin). The lack of the biologically active tetrahydro form of the cofactor may also cause hyperphenylalaninaemia.

\section{MATERIALS AND METHODS}

Pterin standards were purchased from Dr B. Schircks Laboratory (Jona, Switzerland). Dehydratase-free rat liver phenylalanine hydroxylase was purified according to the method of Shiman (1987), followed by two further purification steps: ion-exchange chromatography (10 ml DEAE Toyoaperl 650M) and gel filtration (Sephacryl S$200 \mathrm{HR}$, column $1.6 \times 100 \mathrm{~cm}$ ) (Rebrin, unpublished). Catalase and NADH were purchased from Boehringer (Mannheim, Germany); dihydrofolate reductase, dihydropteridine reductase (DHPR) from Sigma (St Louis, MO, USA).

The pterins, previously oxidized with manganese dioxide under acidic conditions, were measured by HPLC with fluorescence detection (excitation/emission wavelengths were $350 / 450 \mathrm{~nm}$ ) (Niederwieser et al 1982).

Inhibition experiments with dehydratase-free rat liver phenylalanine hydroxylase were performed in $0.1 \mathrm{~mol} / \mathrm{L}$ Tris- $\mathrm{HCl}$, with $0.35 \mathrm{U}$ enzyme (specific activity $8.5 \mathrm{U} / \mathrm{mg}$ protein), $1 \mathrm{mmol} / \mathrm{L}$ L-phenylalanine, and an excess of both catalase and DHPR, 
$5 \mathrm{mmol} / \mathrm{L} \mathrm{NADH}$, L-tetrahydrobiopterin $\left(\mathrm{L}^{-} \mathrm{BH}_{4}\right)$ as substrate (concentrations from 5 to $20 \mu \mathrm{mol} / \mathrm{L}$ ) and $\mathrm{L}-7-\mathrm{BH}_{4}$ as potential inhibitor (concentrations from 2 to $30 \mu \mathrm{mol} / \mathrm{L})$.

\section{RESULTS AND DISCUSSION}

The metabolic origin of 7-substituted pterins (7-biopterin and 7-neopterin) in patients with hyperphenylalaninaemia has been a puzzle since their discovery, and only recently a biochemical pathway for their formation has been proposed (Curtius et al 1989; Adler 1991). The fact that loading of patients suffering from primapterinuria with $\mathrm{L}_{-} \mathrm{BH}_{4}$ leads to an increase of biopterin and 7-biopterin (primapterin) (Curtius et al 1990b), and the finding that the stereochemistry of the side-chain of biopterin and primapterin is the same (Viscontini 1990), is in agreement with the proposal that 7-pterins originate from their 6-isomers. As an explanation for the observed transient hyperphenylalaninaemia one might speculate that the abnormal metabolites negatively affect the phenylalanine hydroxylase activity and that this leads to an increase of the plasma phenylalanine.

Along this line, kinetic studies carried out with phenylalanine hydroxylase using 7-(L-erythro-1',2'-dihydroxypropyl)pterin (7-tetrahydrobiopterin $\left.=\mathrm{L}-7-\mathrm{BH}_{4}\right)$ showed that this pterin is both a substrate and a competitive inhibitor of phenylalanine hydroxylase. As expected, the inhibition experiments demonstrated that $7-\mathrm{BH}_{4}$ competes with $6-\mathrm{BH}_{4}$ for the active site of the phenylalanine hydroxylase. The estimated $K_{\mathrm{i}}$ value of $8 \mu \mathrm{mol} / \mathrm{L}$ for $7-\mathrm{BH}_{4}$ compares to $K_{\mathrm{m}}$ values of $20 \mu \mathrm{mol} / \mathrm{L}$ and $1.5 \mu \mathrm{mol} / \mathrm{L}$ for $7-\mathrm{BH}_{4}$ and $6-\mathrm{BH}_{4}$ as substrates. This observation strongly suggests that in these patients phenylalanine hydroxylase inhibition by 7 -substituted pterins, causes or contributes to hyperphenylalaninaemia.

Since in these patients hyperphenylalaninaemia is transient, one can also speculate that in different stages of development, in addition to $\mathrm{L}_{-} \mathrm{BH}_{4}$, other pterin derivatives, e.g. tetrahydroneopterin, may act as cofactors for phenylalanine hydroxylase. Upon incubation of D-dihydroneopterin with dihydrofolate reductase we found high concentrations of tetrahydroneopterin. Since in vitro tetrahydroneoptrin is a cofactor for phenylalanine hydroxylase, and in spite of the fact, that the activity is only $20 \%$ compared with $\mathrm{BH}_{4}$, this molecule might play an important role in 6-pyruvoyl tetrahydropterin synthase-deficient patients. Since these patients excrete high neopterin but very low biopterin levels, D-tetrahydroneopterin might thus act as cofactor for the aromatic amino acid hydroxylases. Here we thus have a possible explanation for the observation that, while in these patients the levels of neurotransmitters are reduced, they are not abolished.

Using 6-hydroxymethyltetrahydropterin for the in vitro incubation with phenylalanine hydroxylase free of carbinolamine dehydratase, we also observed formation of 7 hydroxymethyltetrahydropterin. This observation suggests that during the phenylalanine hydroxylase reaction conversion of 6- and 7-isomers via the carbinolamine is a general process.

To obtain conclusive information about the in vivo situation in primapterinuric patients, including those suffering from the suspected pterin-4a-carbinolamine dehydratase deficiency, 4a-carbinolamine dehydratase activity ought to be measured 
directly in the tissue of the patients. Additionally conversion of $6-\mathrm{BH}_{4}$ to $7-\mathrm{BH}_{4}$ should be demonstrated also in vivo. For this purpose, side-chain deuterium-labelled L-6- $\mathrm{BH}_{4}$ was synthesized in our group (Adler et al 1990). This substance will be administered to the patients in order to follow the fate of the $\mathrm{L}_{\mathrm{B}} \mathrm{BH}_{4}$ side-chain. In conclusion, and based on our recent results, the pathway of the phenylalanine hydroxylase reaction and the role of the enzymes involved can be formulated as shown in Figure 1.

\section{ACKNOWLEDGEMENTS}

This work was supported financially by the Swiss National Science Foundation, project No. 31-28797.90.

\section{REFERENCES}

Adler C, Curtius H-Ch, Datta S, Viscontini M (1990) A simple synthesis of 5-deoxy-L$\left[5-{ }^{2} \mathrm{H}_{1}\right]$ arabinose and $\mathrm{L}-\left[3^{\prime}-{ }^{2} \mathrm{H}_{1}\right]$ biopterin. Helv Chim Acta 73: $1058-1063$.

Adler C, Curtius H-Ch, Pfleiderer W (1991) Theoretical considerations regarding the synthesis of 7-substituted pterins. In Blau N, Curtius H-Ch, Levine RA, Cotton RGH, eds. Pterins and Biogenic Amines in Neurology, Pediatrics and Immunology. Grosse Pointe, MI: Lakeshore Publishing Co., 69-72.

Curtius H-Ch, Kuster T, Matasovic A, Blau N, Dhondt J-L (1988) Primapterin, anapterin, and 6-oxo-primapterin, three new 7-substituted pterins identified in a patient with hyperphenylalaninemia. Biochem Biophys Res Commun 153: 715-721.

Curtius H-Ch, Adler C, Matasovic A, Akino M (1989) 7-Substituted pterins, a new class of mammalian pteridines. In Curtius H-Ch, Ghisla S, Blau N, eds. Chemistry and Biology of Pteridines. Berlin: Walter de Gruyter, 274-285.

Curtius H-Ch, Kuster T, Matasovic A et al (1990a) 7-Substituted pterins. A new class of mammalian pteridines. $J$ Biol Chem 265: 3923-3930.

Curtius H-Ch, Adler C, Rebrin I, Heizmann CW, Ghisla S (1990b) 7-Substituted pterins: formation during phenylalanine hydroxylation in the absence of dehydratase. Biochem Biophys Res Commun 172: 1060-1066.

Davis MD, Kaufman S, Milstien S (1991) Conversion of 6-substituted tetrahydropterins to 7isomers via phenylalanine hydroxylase-generated intermediates. Proc Natl Acad Sci USA 88: $385-389$.

Dhondt J-L, Guibaud P, Rolland MO et al (1988) Neonatal hyperphenylalaninemia presumably caused by a new variant of biopterin synthetase deficiency. Eur J Pediatr 147: 153-157.

Niederwieser A, Staudenmann W, Wetzel E (1982) Automatic HPLC of pterins with and without column switching. In Wachter $\mathrm{H}$, Curtius $\mathrm{H}-\mathrm{Ch}$, Pfleiderer W, eds. Biochemical and Clinical Aspects of Pteridines. Berlin: Walter de Gruyter, 81-102.

Shiman R (1987) Purification and assay of rat liver phenylalanine 4-monooxygenase. Methods Enzymol 142: 17-27.

Viscontini M (1990) Natural primapterin belongs to the L-series of 7-(polyhydroxypropyl)pterins. Helv Chim Acta 73: 1064-1067. 